\title{
Model and Analysis of Demand-side Response Considering Distributed Generation
}

\author{
Nan $\mathrm{Li}^{1, \mathrm{a}}$, Shouzhen Zhu ${ }^{2, \mathrm{~b}}$, Jinghong Zheng ${ }^{2, \mathrm{c}}$ \\ ${ }^{1}$ China Electric Power Research Institute, Beijing, 100192, China \\ ${ }^{2}$ Department of Electrical Engineering, Tsinghua University, Beijing, 100084, China \\ aemail: linanyz@163.com, bemail:zsz@tsinghua.edu.cn, cemail: jhzheng@tsinghua.edu.cn
}

\section{Keywords: Demand-side Response; Distributed Generation; TOU Price}

\begin{abstract}
Rational peak-valley TOU price can change consumer power consumption mode effectively and assure reliable and high efficient operation of power system. Large-scale promotion of distributed generation will have a direct impact on the distribution network and the demand-side response will be faced with new challenges. Based on TOU price, on the premise of ensuring users and distribution companies have a win-win relationship, this paper develop a demand side response integrated model taking into account of distributed generations. Taking IEEE 33-node distribution system for example, analyzes the impact of DG on the load curve and TOU price setting and gives some reasonable advice.
\end{abstract}

\section{Introduction}

The energy crisis and environmental degradation is the urgent problems to be solved. Renewable energy-based distributed generation (DG) is considered to be an important energy production in future. DG can make full use of available dispersed energy, and greatly improve the efficiency of energy utilization [1]. Access of lots of DG will change the power consumption ways, and users should be encouraged to participate in the activities to achieve economic operation, improve system reliability of power supply and improve their efficiency, so there is an urgent need to study the model of demand side response (DR) under the new situation.

DR refers to that users change their conventional power consumer habits according to price signals or incentives to [2]. In the 1970s, the United States established demand side management mechanism to cope with the energy crisis. To the early 2000s, in response to the California electricity crisis, DR mechanism and the electricity market bid auction mechanism were established [3]. Literature [4] summarized implementation methodology of DR and made recommendations for the development of China. Literature [5] proposed the interruptible load mode of agreement, which was based on user behavior, and could guide the user to voluntarily disclose the information about true cost of power shortage. Literature [6] adopted user satisfaction as one of the goals; analyzed responses of different types of loads to price changes based on the electricity price elasticity.

Demand-side response model in this paper is based on peak-valley TOU price. The problems DR will faces under the new situation of high permeation of DG are studied, and a new integrated DR model consideration of DG is proposed. The role of DG to DR is analyzed though IEEE 33 node distribution system and the influence of related parameters of DG to DR are simulated.

\section{Demand side response based on TOU}

TOU mechanism is one of the most popular dynamic time pricing mechanisms. For those users who have installed smart meters, a day can be divided into peak, flat, valley periods. In general, there are two main forms of users' response to the price, namely, single and multi-time period response. Single period response means that how much power users consume only depend on price at that time. Multi-period response refers that the load is transferred from the high price periods to low price periods, so it is not only depend on the price at that time, but also on other periods of price. 
Single and multi- period responses to the electricity price are as in equation (1) and (2).

$d(i)=d_{0}(i) \times\left\{1+\frac{\varepsilon_{i i} \times\left[\rho(i)-\rho_{0}(i)\right]}{\rho_{0}(i)}\right\}$

$d(i)=d_{0}(i)+\sum \varepsilon_{i j} \times \frac{d_{0}(i)}{\rho_{0}(j)} \times\left[\rho(j)-\rho_{0}(j)\right]$

Where, $\rho_{0}$ is the original price; $\rho$ is the TOU price; $d_{0}$ and $d$ are electricity demand before and after DR; $\varepsilon_{i i}$ is the self-elasticity coefficient; $\varepsilon_{i j}$ is the cross-elasticity coefficient; $i$ and $j$ are different time periods.

The load peak and off-peak difference and the maximum load in daily load curve are the main parameters to measure TOU pricing model, namely the optimization objective function are:

$$
\begin{aligned}
& f_{1}=\min \left[\max _{1 \leq t \leq 24} P\left(\rho_{f}, \rho_{p}, \rho_{g}\right)\right] \\
& f_{2}=\min \left[\max _{1 \leq t \leq 24} P\left(\rho_{f}, \rho_{p}, \rho_{g}\right)-\min _{1 \leq t \leq 24} P\left(\rho_{f}, \rho_{p}, \rho_{g}\right)\right]
\end{aligned}
$$

Where, $\rho_{f}, \rho_{p}$ and $\rho_{g}$ respectively denote peak price, flat price and valley price. Constraints besides power balance equations include:

(1) Implement of DG results in reduction of the maximum load, so the distribution company will purchase less power and reserve capacity, and the construction investment associated with the maximum load will be reduced. To encourage the distribution company to participate in DR actively, the distribution company should make a profit no less than the profit before TOU, namely

$$
\begin{aligned}
& \left(\rho_{f} Q_{f}+\rho_{p} Q_{p}+\rho_{g} Q_{g}\right)-\rho_{n}\left(Q_{f}+Q_{p}+Q_{g}\right)-\lambda\left(\max (P)-\max \left(P_{0}\right)\right)-\sum_{t=1}^{T} \rho_{n}^{\prime}\left(b_{n}(t)\right) \geq\left(\rho_{0}-\rho_{n}\right) Q_{0}-\sum_{t=1}^{T} \rho_{n}^{\prime}\left(b_{n 0}(t)\right) \\
& b_{n 0}(t)=r \% P_{0}(t) \\
& b_{n}(t)=r \% P(t)
\end{aligned}
$$

Where, $Q_{f}, Q_{p}$ and $Q_{g}$ respectively denote the electric quantity the distribution company purchases from the superior grid in peak, flat and valley periods; $P_{0}$ and $P$ respectively stand for daily load demand before and after $\mathrm{DR} ; \rho_{n}$ and $\rho_{n}^{\prime}$ respectively denote the distribution company purchases for power and reserve capacity; $\lambda$ is investment coefficient of the distribution company; $Q_{0}$ and $\rho_{0}$ respectively denote for users' daily electricity quantity and the price before DR; $b_{n 0}(t)$ and $b_{n}(t)$ respectively stand for the spare capacity the distribution company purchases in the period $t$ before and after DR; $r \%$ is the proportion of spare capacity.

(2) Users' expenses on power should be less than before DR, that is

$\rho_{f} Q_{f}+\rho_{p} Q_{p}+\rho_{g} Q_{g} \leq \rho_{0} Q_{0}$

(3) Taking into account the existence of the irreducible load which is unaffected by price, assume that the proportion of the irreducible load is $\eta \%$.

\section{Integrated DR model including DG}

DG has an influence on DR models and strategies. Research in this area is scarce. Compared with the traditional DR, new model should consider the following questions:

(1)There are a wide range of loads in distribution network and only parts of them have installed DG. If the capacity of DG is more than the user needs, the redundant power should sell to the distribution company. Therefore a reasonable feed-in tariff of DG is needed.

(2)It needs to count up how much power of DG can sell to the grid company, which has a relationship with the number of users to install DG and the installed capacity.

(3) Overall capacity of DG should be in line with national policy. According to the national grid for distributed power access requirements, the total capacity of the intermittent power should be within $25 \%$ of the maximum permissible load of the superior transformer.

Assuming the permeability of wind power, photovoltaic power and gas unit is $\bar{s}=\left[s_{w g}, s_{p v}, s_{g t}\right]$, 
and the feed-in tariff is $\rho_{D G}$, the objective function of integrated model of DR is as follows:

$$
\begin{aligned}
& f_{1}=\min \left[\max _{1 \leq t \leq 24} P\left(\rho_{f}, \rho_{p}, \rho_{g}, \rho_{D G}, \bar{s}\right)\right] \\
& f_{2}=\min \left[\max _{1 \leq t \leq 24} P\left(\rho_{f}, \rho_{p}, \rho_{g}, \rho_{D G}, \bar{s}\right)-\min _{1 \leq t \leq 24} P\left(\rho_{f}, \rho_{p}, \rho_{g}, \rho_{D G}, \bar{s}\right)\right]
\end{aligned}
$$

The remarkable different from the traditional model is that the electricity quantity users purchases is not the same with the distribution company purchases from the superior grid because of DG. In new model, constraint equation (4) and (7) becomes:

$$
\begin{aligned}
& \left(\rho_{f} q_{f}+\rho_{p} q_{p}+\rho_{g} q_{g}\right)-\lambda\left(\max (P)-\max \left(P_{0}\right)\right)-\rho_{D G} Q_{n e t}-\rho_{n}\left(Q_{f}+Q_{p}+Q_{g}\right)-\sum_{t=1}^{T} \rho_{n}^{\prime}\left(b_{n}(t)\right) \\
& \geq\left(\rho_{0}-\rho_{n}\right) Q_{0}-\sum_{t=1}^{T} \rho_{n}^{\prime}\left(b_{n 0}(t)\right) \\
& \rho_{f} q_{f}+\rho_{p} q_{p}+\rho_{g} q_{g}-\rho_{D G} Q_{n e t} \leq \rho_{0} Q_{0}
\end{aligned}
$$

Where, $q_{f}, q_{p}$ and $q_{g}$ respectively denote electricity quantity users purchases from the distribution company at peak, flat and valley periods; $Q_{n e t}$ is the daily feed-in electricity quantity of all DG.

\section{Test results}

IEEE 33-node distribution system is simulated in this paper, which is shown in Fig.1. The maximum load is $3715 \mathrm{~kW}$ while the minimum load is $2851.1 \mathrm{~kW}$. The Electricity price before DR is $0.56 ¥ / \mathrm{kWh}$. Assuming the distribution company need to buy 5\% of the forecast load as spare capacity at each period. Investment coefficient of the distribution company for a day is $0.1 Y / \mathrm{kW}$.

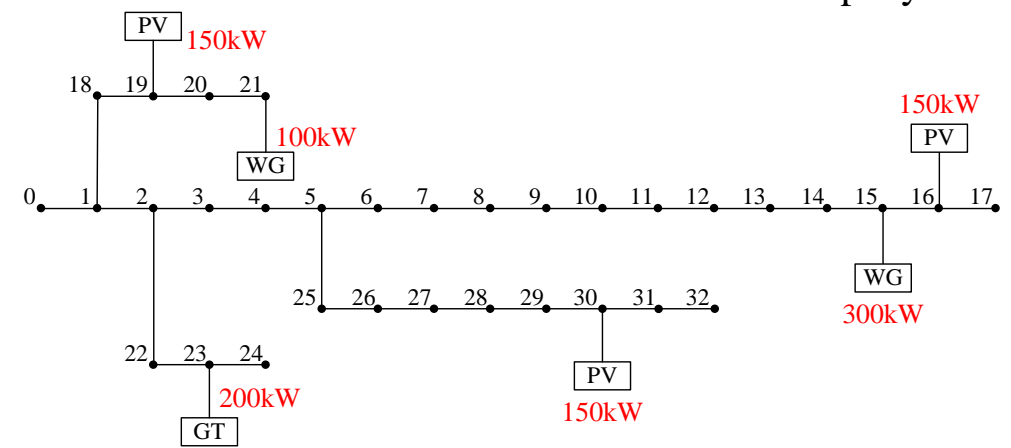

Fig.1. IEEE 33-node distribution system

Though the method of semi-trapezoidal membership function, the time division is as follows: peak periods are 11:00 12:00 and 17:00: $00 \sim$ 21:00 , total of 7 hours; flat periods are 1: 00,9: $00 \sim$ 10:00,13: $00 \sim 16: 00$ and 22: $00 \sim 24: 00$, total of 10 hours; valley periods are 2:00 - 8:00, total of 7 hours. Under different cases, the load response curves after DR are shown in Figure 2, and Table 2 shows the corresponding TOU price.

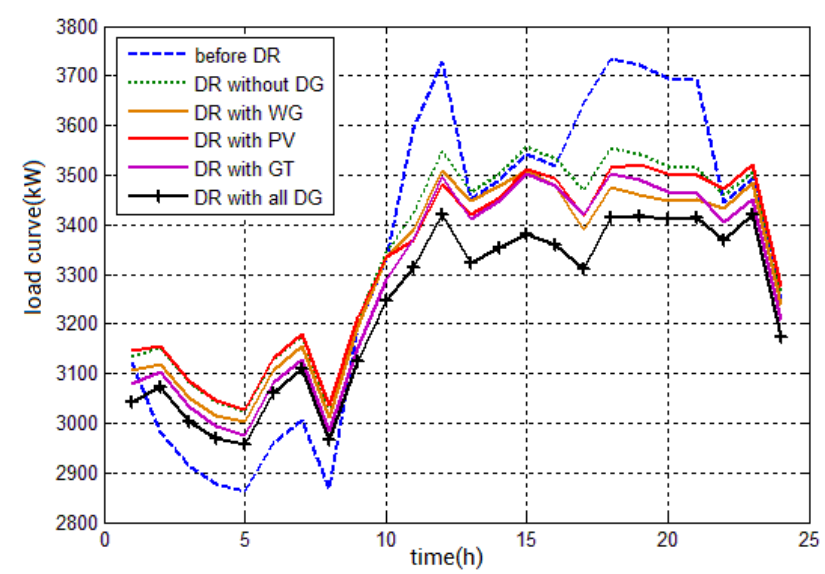

Fig.1. Optimal load curve under different cases of DG 
Table 2. TOU price and load parameters under different cases of DG( $¥ / \mathrm{kWh}, \mathrm{kW})$

\begin{tabular}{|c|c|c|c|c|c|}
\hline & $\begin{array}{c}\text { DR with } \\
\text { DG }\end{array}$ & $\begin{array}{c}\text { DR with } \\
\text { WG }\end{array}$ & $\begin{array}{c}\text { DR with } \\
\text { PV }\end{array}$ & $\begin{array}{c}\text { DR with } \\
\text { GT }\end{array}$ & $\begin{array}{c}\text { DR with } \\
\text { all DG }\end{array}$ \\
\hline peak price & 0.8439 & 0.8785 & 0.8722 & 0.8553 & 0.8745 \\
\hline Flat price & 0.5830 & 0.5749 & 0.5715 & 0.5954 & 0.6072 \\
\hline Valley price & 0.2 & 0.2 & 0.2 & 0.2 & 0.2 \\
\hline The maximum load & 3556.8 & 3507.6 & 3520.8 & 3502.7 & 3419.8 \\
\hline The load peak and off-peak difference & 532.0 & 505.2 & 492.7 & 526.0 & 463.6 \\
\hline
\end{tabular}

We can see that implementation of TOU decreases the peak load and increases the valley load. Participation of DG make significant effect on DR, especially when the wind turbine generators, the solar photovoltaic and the gas turbine units operate simultaneously, the peak load is greatly reduced.

\section{Conclusion}

This paper studies the impact of DG on DR in the situation that DG is evolving at an increasing rate and proposed the integrated DR model. We conclude that development of DG will decrease the electricity quantity the distribution company purchases from the superior grid and reduce the distribution network construction cost. Along with the development of DG in the distribution system, DG is becoming a new mean for users’ participation in DR.

\section{Acknowledgement}

In this paper, the research was sponsored by National High Technology Research and Development of China (863 Program) (Project No. 2014AA051902) and National Basic Research Program of China (973 Program) (Project No. 2013CB228203).

\section{References}

[1] Ma Yiwei, Yang Ping, Guo hongxia, et al. Power source planning of wind-PV-biogas renewable energy distributed generation system[J]. Power System Technology, 2012, 36(9) : 9-14.

[2] Kamalanath Samarakoon, Janaka Ekanayake, Nick Jenkons. Reporting available demand response[J]. IEEE Transactions on Smart Grid, 2013, 4(4) : 1842-1851.

[3] Duy Thanh Nguyen, Michael Negnevitsky, Martin de GRoot. Pool-based demand response exchange - concept and modeling[M]. IEEE Transactions on Smart Grid, 2011, 26(3) : 1677-1685.

[4] Wu Weihua, Pang Jianjun, Chen Guangkai, et al. Research on development of electric power demand side response[J]. Electronic Test, 2014, 3: 86-94.

[5] Wei Linlin, Huang Minxiang. Subdivided user's new mode of interruptible agreement[J]. Electric technology, 2004, 5: 18-20.

[6] Li Hui, Kang Chongqing, Xia Qing. Price based decision making for demand side management considering customer satisfaction index[J]. Power System Technology, 2004, 28(23) : 1-6. 